\title{
Correlation of Computed Tomography Guided Visceral Adiposity \& Sagittal Abdominal Diameter with Atherogenic Index of Plasma in Type 2 Diabetic Patients
}

\author{
Pradyumna Rao ${ }^{1}$, Chavala Mohan Bindu르, Usha Setty Muthihar Ramachary ${ }^{3}$ \\ 1,2,3 Department of Biochemistry, Rajarajeswari Medical College and Hospital, Mysore Rd., \\ Kengeri Satellite Town, Kambipura, Bangalore, Karnataka, India.
}

\section{ABSTRACT}

\section{BACKGROUND}

Type 2 diabetic patients are at high risk for many cardiovascular diseases which are associated with obesity and abdominal fat that includes visceral adipose tissue (VAT) and subcutaneous adipose tissue (SAT). Visceral adiposity contributes to impairment of insulin resistance which is a prerequisite for diabetes and dyslipidaemia. Computed tomography (CT) scan is the gold standard for measurement of VAT but due to its limitations, sagittal abdominal diameter (SAD) is considered which also has a strong correlation with VAT. There is a good relation between VAT and isolated lipid parameters. However, lipid ratios, such as Atherogenic Index of Plasma (AIP) are of higher predictive value for cardiovascular risk. The present study is focused on exploring the relationship between CT guided visceral adiposity and sagittal abdominal diameter with AIP in type 2 diabetic patients.

\section{METHODS}

This study was carried out with a study group of 53 type 2 diabetes patients aged between 25 to 65 years, incorporating appropriate inclusion and exclusion criteria in a tertiary care hospital. The biochemical assays done include triglycerides, HDLcholesterol and blood glucose by standard techniques. AIP was calculated by taking the logarithmic ratio of triglyceride and HDL-cholesterol levels. Measurement of visceral adiposity was done by SAD and CT scan.

\section{RESULTS}

A total of 53 patients with type II diabetes mellitus (DM) were recruited for the study comprising of 35 males and 18 females. Mean age of the patients was $41.717 \pm 11.658$ years. Mean value of SAD was $22.755 \pm 2.70$. Mean value of AIP was $0.164 \pm 0.15$. Mean value of CT measured visceral adiposity was - $77.875 \pm 24.54$ HU. Pearson's correlation coefficient for the relationship between SAD and AIP was 0.741 and Pvalue was less than 0.0001 indicating a positive correlation. Pearson's correlation coefficient for the relationship between CT measured visceral adiposity and AIP was 0.765 and $\mathrm{P}$-value was less than 0.0001 which also shows a positive correlation.

\section{CONCLUSIONS}

In our study we demonstrated that SAD and CT guided visceral adiposity was correlating with AIP in patients with type 2 diabetes mellitus.

\section{KEY WORDS}

Visceral Adiposity, Sagittal Abdominal Diameter, Atherogenic Index of Plasma, Diabetes Mellitus, Computed Tomography Scan
Corresponding Author: Dr. Chavala Mohan Bindu, Department of Biochemistry, 8 D East, Klassic Benchmark Apartments, Bannerghatta Road, Bangalore - 560076,

Karnataka, India.

E-mail: bindu_bcm@yahoo.co.in

DOI: $10.14260 / j e m d s / 2021 / 244$

How to Cite This Article:

Rao $P$, Bindu CM, Ramachary USM. Correlation of computed tomography guided visceral adiposity \& sagittal abdominal diameter with atherogenic index of plasma in type 2 diabetic patients. J Evolution Med Dent Sci 2021;10(16): 1145-1149, DOI: $10.14260 /$ jemds/2021/244

Submission 04-12-2020,

Peer Review 20-02-2021,

Acceptance 26-02-2021,

Published 19-04-2021.

Copyright (C) 2021 Pradyumna Rao et al. This is an open access article distributed under Creative Commons Attribution License [Attribution 4.0 International (CC BY 4.0)] 


\section{BACKGROUND}

The worldwide prevalence of obesity and obesity related diseases continues to rise. The number of people with diabetes in India increased from 26.0 million in 1990 to 65.0 million in 2016. The prevalence of diabetes in adults aged 20 years or older in India increased from $5.5 \%$ in 1990 to $7.7 \%$ in $2016 .{ }^{1}$

Diabetic patients are at high risk for many cardiovascular disorders. These include coronary heart disease, stroke, peripheral arterial disease, cardiomyopathy, and congestive heart failure. The leading causes of diabetes related morbidity and mortality are now cardiovascular complications. In patients with diabetes, the public health impact of cardiovascular disease (CVD) is huge and is increasing. ${ }^{2}$

Type 2 diabetes mellitus and cardiovascular diseases are associated with obesity and abdominal fat and is strongly associated with both the above conditions. Abdominal fat includes subcutaneous adipose tissue and visceral adipose tissue compartments. ${ }^{3}$ Visceral adipose tissue is now recognised to have metabolic, endocrine, and immune functions and has a stronger correlation to obesity related disorders than SAT. 4,5

Increased visceral adiposity, through an increased fatty acid production and secretion of higher concentrations of inflammatory adipocytokines contribute to the impairment of insulin signalling pathway resulting in insulin resistance which is a prerequisite for diabetes, dyslipidaemia and cardiovascular disease with a risk of early death.6,7

Computed tomography is considered as the "gold standard" for the measurement of visceral fat. However, CT scans are costly, involve exposure to ionizing radiation, and may not be universally available. ${ }^{8}$ Due to these limitations, much effort has been made to find inexpensive, easily obtainable anthropometric measures such as sagittal abdominal diameter which has strong correlation to the amount of VAT. ${ }^{9}$

Studies have shown a good correlation between VAT and isolated lipid parameters, including triglyceride levels and HDL-cholesterol. ${ }^{10}$ However, it has been found that lipid ratios are of higher predictive value for cardiovascular risk, as they reflect the interactions between different lipoprotein fractions. One such entity, namely, AIP, is the logarithmic ratio of TG / HDL is one of the biochemically significant ratios. ${ }^{11}$ Due to its strong correlation with lipoprotein particle size, it has been accepted as a highly predictive entity. ${ }^{12}$ Patients who have type 2 diabetes mellitus have high visceral fat content and this is associated with AIP. ${ }^{13}$

In this study, we intend to investigate the strength of association (correlation) of SAD and CT guided visceral adiposity with AIP in patients with type 2 diabetes mellitus.

\section{METHODS}

This was a cross sectional study conducted among 53 type 2 diabetes patients ( 25 to 65 years age group) in a tertiary care hospital from July 1st, 2018 to August 25th, 2018.

\section{Exclusion Criteria}

- Patients with history of familial dyslipidaemias.

- Patients who were on treatment with hypolipidemic drugs.

- Patients suffering from thyroid disease.

- Patients with history of alcohol abuse.

\section{Data Collection and Processing}

1. Fasting blood sample (7 $\mathrm{ml}$ of venous blood) was collected from the study group under aseptic precaution after obtaining informed consent for analysis in the below mentioned tubes

- Fluoride containing n-vac grey capped tube for the estimation of blood glucose.

- Clot activator containing n-vac red capped tube for the estimation of triglycerides (TGs) and HDLcholesterol.

The biochemical parameters were estimated using the following methods:

- Blood glucose by glucose oxidase enzymatic method.

- HDL-cholesterol by immuno-inhibition method.

- Triglycerides (TGs) by glycerol-3-phosphate oxidase method.

2. AIP was calculated by taking the logarithmic ratio of triglyceride level and HDL-cholesterol level using the values obtained from the above investigations.

AIP $=\log$ (TG / HDL-cholesterol).

3. Sagittal abdominal diameter or abdominal height was measured after normal expiration to the nearest $0.1 \mathrm{~cm}$ in supine position with straight legs on a firm examination table, without clothes in the measurement area. At the level of iliac crest, SAD was measured using a ruler and water level, mean SAD was calculated as the average of 3 trials of the measurement of SAD.

4. Measurement of visceral adiposity was done by computed tomography at the level of the umbilicus after deep inspiration (approximately the level of L4 and L5) with attenuation range of - 190 to - $30 \mathrm{HU}$ (Hounsfield units) using Siemens SOMATOM Perspective 128 slices Multidetector CT scanner.

\section{Statistical Analysis}

Data was collected and analysed using SPSS version 20 and MS Excel. Correlation of SAD and CT measured visceral adiposity with AIP in diabetic patients using Pearson's correlation coefficient and the statistical significance was taken as Pvalues less than 0.05 .

\section{RESULTS}

A total of 53 patients with type II DM were recruited for the study comprising of 35 males and 18 females, to correlate visceral adiposity guided by CT and SAD with AIP in the stipulated time frame (8 weeks' duration) after obtaining ethical clearance from the ethical review board of the 
institution. Mean age of the patients recruited for the study was $41.717 \pm 11.658$ years. [Table 1 ]

\begin{tabular}{|ccc|}
\hline Age (in years) & No. $(\mathbf{N}) \mathbf{= 5 3}$ & Percentage $\%$ \\
$<30$ & 8 & 15.09 \\
$31-40$ & 23 & 43.40 \\
$41-50$ & 6 & 11.32 \\
$51-60$ & 14 & 26.42 \\
$>60$ & 2 & 3.77 \\
Mean age \pm SD & $41.717 \pm 11.658$ \\
Gender & 35 & 66.04 \\
Male & 18 & 33.96 \\
\hline Female & \\
\hline Table 1. Age and Gender Distribution of the Subjects \\
\hline
\end{tabular}

\begin{tabular}{|cc|}
\hline Parameter & Mean \pm 2 Standard Deviations \\
(SD) \\
SAD in cm & $22.755 \pm 2.70$ \\
AIP & $0.164+0.15$ \\
CT measured visceral adiposity (in HU) & $-77.875 \pm 24.54$ \\
Table 2. Mean and Standard Deviation of SAD, \\
AIP and CT Measured Visceral Adiposity \\
\hline
\end{tabular}

\begin{tabular}{|ccc|}
\hline & $\begin{array}{c}\text { Pearson's Correlation } \\
\text { Coefficient }\end{array}$ & P-Value \\
\hline SAD and AIP & 0.741 & $<0.0001$ \\
CT measured visceral adiposity and & 0.765 & $<0.0001$ \\
\hline AIP & Table 3. Correlation Coefficients between SAD and AIP \\
and CT Measured Visceral Adiposity and AIP & \\
\hline
\end{tabular}
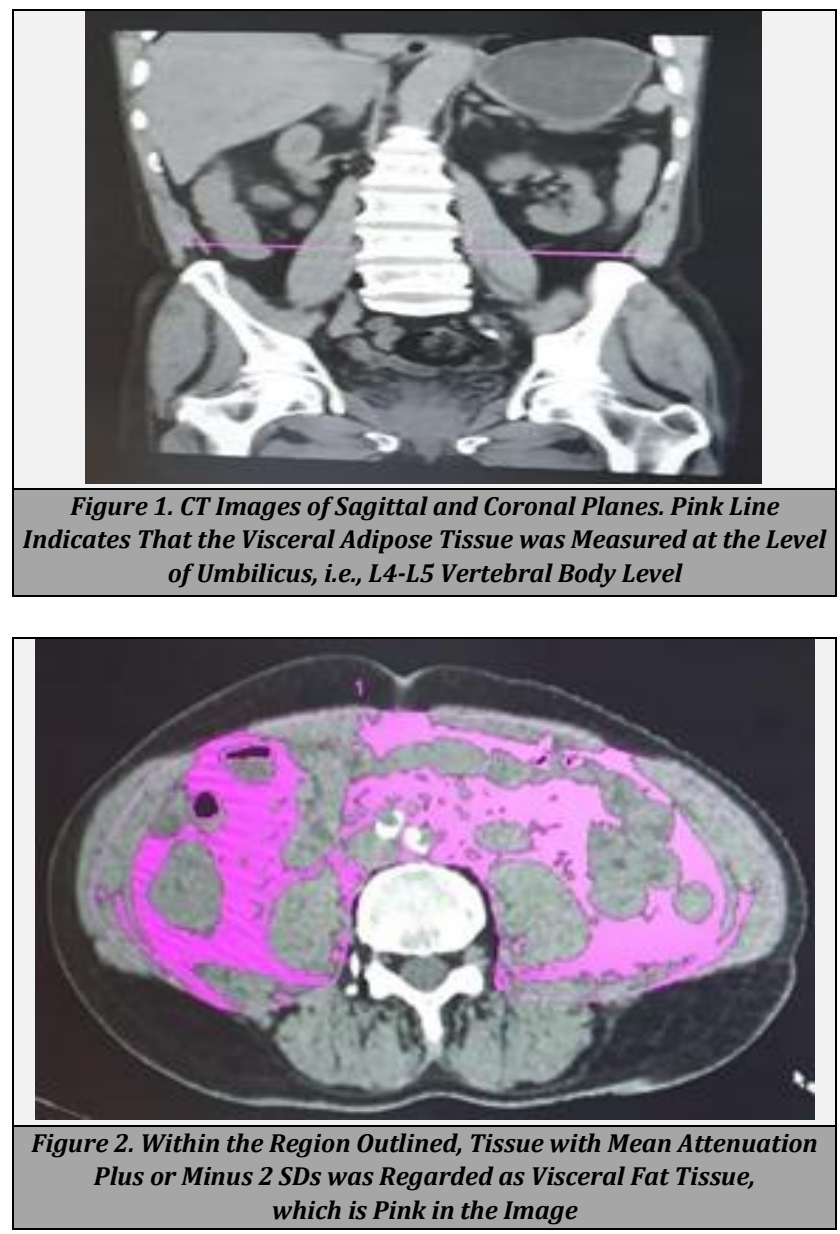

Mean value of SAD was $22.755 \pm 2.70$. Mean value of AIP was $0.164 \pm 0.15$. [Table 2] Mean value of CT measured visceral adiposity \pm 2 SD was $-77.875 \pm 24.54$ HU. Pearson's correlation coefficient for the relationship between SAD and AIP was 0.741 and $P$ value was less than 0.0001. [Table 3]
Pearson's correlation coefficient for the relationship between CT measured visceral adiposity and AIP was 0.765 and $P$ value was less than 0.0001 . [Table 3]

Since the Pearson's correlation coefficient for the association between SAD and AIP was 0.741, we can conclude that there is a significant positive correlation between the 2 entities.
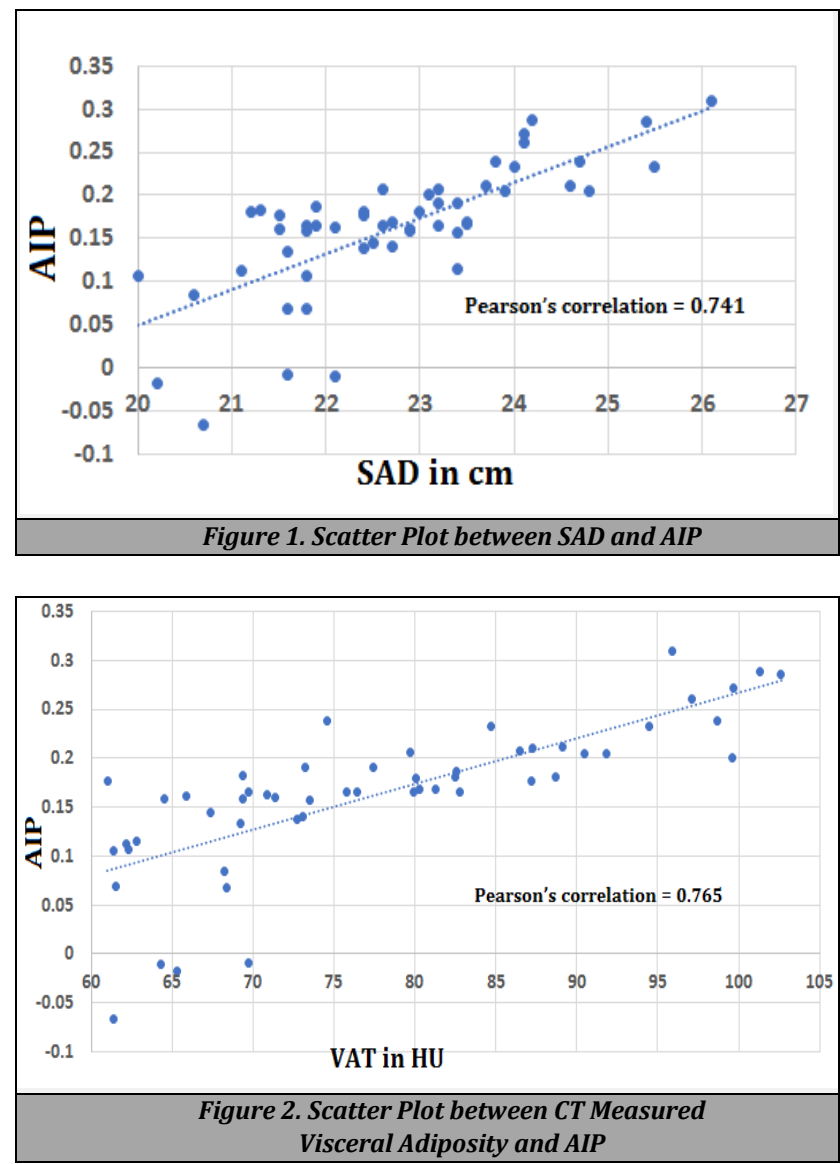

Similarly, the Pearson's correlation coefficient for the association between CT measured visceral adiposity and AIP was 0.765 , suggesting that there is a significant positive correlation between the 2 entities.

\section{DISCUSSION}

In this present study, we have evaluated the association of AIP with SAD and CT guided visceral adiposity in type 2 diabetic patients.

The public health impact of cardiovascular disease in patients with diabetes mellitus is huge and increasing, and such patients are at high risk for many cardiovascular disorders like coronary heart disease, stroke, peripheral arterial disease, cardiomyopathy, and congestive heart failure. $^{2}$

Abdominal fat includes both subcutaneous adipose tissue and visceral adipose tissue compartments. ${ }^{3}$ Visceral adipose tissue has a stronger correlation to obesity related disorders than SAT. It is thought to exhibit this stronger correlation by virtue of its endocrine, metabolic and immune functions. ${ }^{4,5}$ There are several studies which have shown association of 
VAT with cardiometabolic risk. Due to a high rate of lipolysis, free fatty acids are transported to the liver where it could be deposited. This increased intrahepatic fat content is a cardiometabolic risk factor. Furthermore, VAT has a high secretion rate of growth factors, cytokines, and hormones which are in turn involved in the pathogenesis of cardiometabolic diseases. ${ }^{15}$

Dyslipidaemia associated with increased visceral adiposity is a consequence of increased efflux of free fatty acid from adipose tissue leading to increased synthesis of triglycerides, cholesterol and Apo B in the liver resulting in elevated VLDL and LDL in plasma. Finally, cholesteryl ester transfer protein (CETP) mediated exchange of core lipids among the different lipoproteins results in sdLDL (small dense LDL) and low HDL characteristic of atherogenic dyslipidaemia, setting a stage for future cardiovascular events. ${ }^{16}$

In addition, AIP which indicates atherogenic dyslipidaemia is strongly correlated with visceral adiposity. ${ }^{12}$ Visceral adiposity can be measured by SAD and by using computed tomography. As discussed by Jeong Yoon Yim, Donghee Kim, and Sang-Heon Cho, SAD has been proven to be excellent estimate of visceral fat implying it as a good marker of insulin resistance than other anthropometric measures, irrespective of age, sex, and the degree of obesity. ${ }^{17}$

In our study it was found that SAD positively correlated with AIP with correlation coefficient of 0.741 and P value of < 0.0001 implying strong statistical significance. We have also examined the correlation between CT guided VAT and AIP which revealed a correlation coefficient value of 0.765 with statistical significance substantiated with P-value of $<0.0001$. This indicates that both of the above-mentioned entities are independently, strongly and directly related to the AIP, which itself has been accepted as a highly predictive entity of cardiovascular risk. ${ }^{12}$

\section{CONCLUSIONS}

Type 2 diabetes mellitus patients with increased visceral adiposity and high AIP were found to have increased cardiovascular risk and more. AIP has the potential to emerge as a potent, cost effective marker for the evaluation of the risk of heart disease. In this study it has been established that SAD and CT guided visceral adiposity correlates equally and effectively with AIP. SAD being a simple, non-invasive and cost-effective, can be used as an alternative tool for evaluation of cardiovascular risk instead of CT guided visceral adiposity in routine clinical practice.

By carrying out further research in more extensive and broader study groups, we can further cement this principle and can establish sagittal abdominal diameter as an important tool in the evaluation of cardiometabolic risk in the near future.

Data sharing statement provided by the authors is available with the full text of this article at jemds.com.

Financial or other competing interests: None.

Disclosure forms provided by the authors are available with the full text of this article at jemds.com.

\section{REFERENCES}

[1] India State-Level Disease Burden Initiative Diabetes Collaborators. The increasing burden of diabetes and variations among the states of India: the global burden of disease study 1990-2016. Lancet Glob Health 2018;6:e1352-62.

[2] Diabetes mellitus: a major risk factor for cardiovascular disease. A joint editorial statement by the American Diabetes Association; the national heart, lung and blood institute: the Juvenile Diabetes Foundation International: the National Institute Of Diabetes And Digestive And Kidney Diseases: and the American Heart Association. Circulation 1999;100(10):1132-3.

[3] Preis SR, Hwang SJ, Coady S, et al. Trends in all-cause and cardiovascular disease mortality among women and men with and without diabetes mellitus in the Framingham heart study 1950 to 2005. Circulation 2009;119(13):1728-35.

[4] Fox CS, Massaro JM, Hoffmann U, et al. Abdominal visceral and subcutaneous adipose tissue compartments: association with metabolic risk factors in the Framingham Heart Study. Circulation 2007;116(1):3948.

[5] Hayashi T, Boyko EJ, McNeely MJ, et al. Visceral adiposity, not abdominal subcutaneous fat area, is associated with an increase in future insulin resistance in Japanese Americans. Diabetes 2008;57(5):1269-75.

[6] Hamdy 0, Porramatikul S, Al-Ozairi E. Metabolic obesity: between visceral and sub cutaneous fat. Curr Diabetes Rev 2006;2(4):367-73.

[7] Kim SK, Kim HJ, Hur KY, et al. Visceral fat thickness measured by ultra sonography, can estimate not only visceral obesity but also risks of cardio vascular and metabolic diseases. Am J Clin Nutr 2004;79(4):593-9.

[8] Van Der Kooy K, Seidell JC. Techniques for the measurement of visceral fat: a practical guide. Int J Obes Relat Metab Disord 1993;17(4):187-96.

[9] Frenhani PB, Pimentel GD, Portero-Mclellan KC, et al. Sagittal abdominal diameter as a predictor of visceral abdominal fat, insulin resistance, dyslipidaemia and inflammation in overweight Brazilian adults. Clinical Nutrition Supplements 2008;3(Suppl 1):126.

[10] Sam S, Haffner S, Davidson M, et al. Relationship of abdominal visceral and subcutaneous adipose tissue with lipoprotein particle number and size in type 2 diabetes. Diabetes 2008;57(8):2022-7.

[11] Adaja TM, Onovughakpo-Sakpa OE. Atherogenic index of plasma and visceral adiposity index among healthcare workers in University of Benin Teaching Hospital, Benin City, Nigeria. International Journal of Tropical Disease \& Health 2018;29(3):1-11.

[12] Dobiasovia M. AIP--Atherogenic Index of Plasma as a significant predictor of cardiovascular risk: from research to practice. Vnitr Lek 2006:52(1):64-71.

[13] Song P, Xu L, Xu J, et al. Atherogenic index of plasma is associated with body fat level in type 2 diabetes mellitus patients. Curr Vasc Pharmacol 2018;16(6):589-95. 
[14] American Diabetes Association. Diagnosis and classification of diabetes mellitus. Diabetes Care 2010;33(Suppl 1):S62-9.

[15] Elffers TW, De Mutsert R, Lamb HJ, et al. Body fat distribution, in particular visceral fat, is associated with cardiometabolic risk factors in obese women. PLoS One 2017;12(9):e0185403.
[16] Brunzell JD, Hokanson JE. Dyslipidaemia of central obesity and insulin resistance. Diabetes Care 1999;22(Suppl 3):C10-3.

[17] Yim JY, Kim D, Lim SH, et al. Sagittal abdominal diameter is a strong anthropometric measure of visceral adipose tissue in the Asian general population. Diabetes Care 2010;33(12):2665-70. 